\title{
ON STATISTICS INDEPENDENT OF A COMPLETE SUFFICIENT STATISTIC
}

\author{
By D. BASU \\ Indian Statistical Institute, Calcutta
}

1. INTRODUCTION

If $\left\{P_{\theta}\right\}, \theta \in \Omega$, be a family of probability measures on an abstract sample space $\checkmark$ and $T$ be a sufficient statistic for $\theta$ then for a statistic $T_{1}$ to be stochastically independent of $T$ it is necessary that the probability distribution of $T_{1}$ be independent of $\theta$. The condition is also sufficient if $T$ be a boundedly complete sufficient statistic. Certain well-known results of distribution theory follow immediately from the above considerations. For instance, if $x_{1}, x_{2}, \ldots, x_{n}$, are independent $N(\mu, \sigma)$ 's then the sample mean $\bar{x}$ and the sample variance $s^{2}$ are mutually independent and are jointly independent of any statistic $f$ (real or vector valued) that is independent of change of scale and origin. It is also deduced that if $x_{1}, x_{2}, \ldots, x_{n}$, are independent random variables such that their joint distribution involves an unknown location parameter $\theta$ then there can exist a linear boundedly complete sufficient statistic for $\theta$ only if the $x$ 's are all normal. Similar characterizations for the Gamma distribution also are indicated.

\section{Definitions}

Let $(S, \mathscr{A})$ be an arbitrary measurable space (the sample space) and let $\left\{P_{\theta}\right\}$, $\theta \epsilon \Omega$, be a family of probability measures on $\mathcal{A}$.

Definition 1: Any measurable transformation $T$ of the sample space $(\mathfrak{S}, \mathscr{A})$ onto a measurable space $(\mathcal{I}, \mathscr{B})$ is called a statistic. The probability measures on $\mathcal{B}$ induced by the statistic $T$ are denoted by $\left\{P_{\theta}^{T}\right\}, \theta \in \Omega$.

For every $\theta \epsilon \Omega$ and $A \epsilon \mathcal{A}$ there exists an essentially unique real valued $\beta$-measurable function $f_{\theta}(A \mid t)$ on $\mathcal{J}$ such that the equation

$$
P_{\theta}\left(A \cap T^{-1} B\right)=\int_{B} f_{\theta}(A \mid t) d P_{\theta}^{T}
$$

holds for every $B \in \mathcal{B}$. The set of points $t$ for which $f_{\theta}(A \mid t)$ falls outside the closed interval $(0,1)$ is of $P_{\theta}^{T}$-measure zero for every $\theta \in \Omega$. We call $f_{\theta}(A \mid t)$ the conditional probability of $A$ given that $T=t$ and that $\theta$ is the true parameter point.

Definition 2: A statistic $T$ is said to be independent of the parameter $\theta$ if, for every $B \in \mathcal{B}, P_{\theta}^{T}(B)$ is the same for all $\theta \epsilon \Omega$.

Definition 3: The two statistics $T$ and $T_{1}$, with associated measurable spaces $(\mathcal{I}, \mathscr{B})$ and $\left(\mathcal{J}_{1}, \mathscr{B}_{1}\right)$ respectively, are said to be stochastically independent of each other if, for every $B \in B$ and $B_{1} \in \mathscr{B}_{1}$

$$
P_{\theta}\left(T^{-1} B \cap T_{1}^{-1} B_{1}\right) \equiv P_{\theta}\left(T^{-1} B\right) P_{\theta}\left(T_{1}^{-1} B_{1}\right)
$$

for all $\theta \in \Omega$. 
Now,

$$
P_{\theta}\left(T^{-1} B \cap T_{1}^{-1} B_{1}\right) \equiv \int_{B} f_{\theta}\left(T_{1}^{-1} B_{1} \mid t\right) d P_{\theta}^{T^{\prime}} .
$$

It follows, therefore, that a necessary and sufficient condition in order that $T$ and $T_{1}$ are stochastically independent is that the integrand above is essentially independent of $t$, i.e.

$$
f_{\theta}\left(T_{1}^{-1} B_{1} \mid t\right)=P_{\theta}\left(T_{1}^{-1} B_{1}\right)=P_{\theta}^{T_{1}}\left(B_{1}\right)
$$

for all $t \epsilon T$ excepting possibly for a set of $P_{\theta}^{T}$-measure zero.

Definition 4: The statistic $T$ is called a sufficient statistic (Halmos and Savage, 1949 ) if for every $A \in \mathcal{A}$ there exists a function $f(A \mid t)$ which is independent of $\theta$ and which satisfies equation (1) for every $\theta \in \Omega$.

Let $G$ be the class of all real valued, essentially bounded, and $\mathcal{B}$-measurable functions on $\mathcal{Z}$.

Definition 5: The family of probability measures $\left\{P_{\theta}^{T}\right\}$ is said to be boundedly complete (Lehmann and Scheffé, 1950) if for any $g \in G$ the identity

$$
\int_{T} g(t) d P_{\theta}^{T} \equiv 0 \quad \text { for all } \theta \in \Omega
$$

implies that $g(t)=0$ excepting possibly for a set of $P_{\theta}^{T}$-measure zero for all $\theta$. $\left\{P_{\theta}^{T}\right\}$ is called complete if the condition of essential boundedness is not imposed on the integrand in (2). The statistic $T$ is called complete (boundedly complete) if the corresponding family of measures $\left\{P_{\theta}^{T}\right\}$ is so.

\section{Sufficiency AND INDEPENDENCE}

For any two statistics $T_{1}$ and $T$ we have for any $B_{1} \in \mathcal{B}_{1}$

$$
P_{\theta}^{T_{3}}\left(B_{1}\right)=P_{\theta}\left(T_{1}^{-1} B_{1}\right)=\int_{\mathcal{Z}} f_{\theta}\left(T_{1}^{-1} B_{1} \mid t\right) d P_{\theta}^{\mathrm{T}} .
$$

Now if $T$ be a sufficient statistic then the integrand is independent of $\theta$ and if, moreover, $T_{1}$ is stochastically independent of $T$ then the integrand is essentially independent of $t$ also. Thus, the right hand side of (3) is independent of $\theta$ and so we have

Theorem 1: Any statistic $T_{1}$ stochastically independent of a sufficient statistic $T$ is independent of the parameter $\theta$.

That the direct converse of the above result is not true will be immediately apparent if we take for the sufficient statistic $T$ the identity mapping of $(\mathcal{S}, \mathscr{A})$ into itself. No statistic $T_{1}$ independent of $\theta$ will then be stochastically independent of $T$ excepting in the trival situation where $T_{1}$ is essentially equal to a constant. We, however, have the following weaker but important converse.

Theorem 2: If $T$ be a boundedly complete sufficient statistic then any statistic $T_{\mathfrak{x}}$ which is independent of $\theta$ is stochastically independent of $T$. 


\section{STATISTICS INDEPENDENT OF A COMPLETE SUFFICIENT STATISTIC}

Proof: Since $T$ is sufficient the integrand in (3) is independent of $\theta$. It is also essentially bounded. Now the left hand side of (3) is independent of $\theta$ since $T_{1}$ is independent of $\theta$. Hence, from bounded completeness of $\left\{P_{\theta}^{T}\right\}$ it follows that the integrand in (3) is essentially independent of $t$ as well. That is, $T_{1}$ is stochastically independent of $T$.

In the next section we demonstrate how the above theorem may be used to get a few interesting results in distribution theory.

\section{SOME CHARACTERIZATIONS OF DISTRIBUTIONS WITH LOCATION AND SCALE PARAMETERS}

Let $\underset{\sim}{x}=\left(x_{1}, x_{2}, \ldots, x_{n}\right)$ be a random variable in an $n$-dimensional Euclidean space whose probability distribution involves an unknown location parameter $\mu$ and a scale parameter $\sigma>0$. Then any measurable function $f\left(x_{1}, x_{2}, \ldots, x_{n}\right)$ which is independent of change of origin and scale, i.e.

$$
f\left(\frac{x_{1}-a}{b}, \ldots, \frac{x_{n}-a}{b}\right) \equiv f\left(x_{1}, \ldots, x_{n}\right)
$$

for all $a$ and $b>0$ is independent of the unknown parameter $(\mu, \sigma)$. Now, if there exists a boundedly complete sufficient statistic $T$ for $(\mu, \sigma)$ then $f$ must be stochastically independent of $T$. For example, if $x_{1}, x_{2}, \ldots, x_{n}$, are independent observations on a normal variable with mean $\mu$ and s.d. $\sigma$ then it is well known that $T=(\bar{x}, s)$ is a sufficient statistic ( $\bar{x}$ is the sample mean and $s$ the sample s.d.). The completeness of $T$ follows from the unicity property of the bivariate Laplace transform. It then follows from Theorem 2 that any measurable function $g(\bar{x}, s)$ of $\bar{x}$ and $s$ is stochastically independent of any measurable function $f\left(x_{1}, x_{2}, \ldots, x_{n}\right)$ of the observations that is independent of change of origin and scale. The functions $g$ and $f$ need not be real valued. For instance, we may have

$$
g=\left(\Sigma x_{i}^{2}, \sum_{i \ngtr j} x_{i} x_{j}\right)
$$

and

$$
f=\left(\frac{\Sigma\left(x_{i}-\bar{x}\right)^{3}}{s^{3}}, \frac{\Sigma\left(x_{i}-\bar{x}\right)^{4}}{s^{4}}, \ldots\right)
$$

Again the stochastic independence of $\bar{x}$ and $s$ follows from the fact that, for any fixed $\sigma$, the statistic $\bar{x}$ is a complete sufficient statistic for $\mu$ and that $s$, by virtue of its being independent of change of origin, is independent of the location parameter $\mu$.

Now let $x_{1}, x_{2}, \ldots, x_{n}$, be independent random variables with joint d.f. $F_{1}\left(x_{1}-\theta\right), F_{2}\left(x_{2}-\theta\right), \ldots, F_{n}\left(x_{n}-\theta\right)$.* Since $\theta$ is a location parameter it follows that any linear function $\Sigma a_{i} x_{i}$ with $\Sigma a_{i}=0$ is independent of $\theta$. If $\Sigma b_{i} x_{i}$ is a boundedly complete sufficient statistic for $\theta$ then from Theorem 2 it follows that $\Sigma a_{i} x_{i}$ is independent of $\Sigma b_{i} x_{i}$.

* For the sake of notational convenience, we make no distinction between random variables and the values that they may assume. 
Now, since $\Sigma b_{i} x_{i}$ is a sufficient statistic it follows that every $b_{i} \neq 0$. For, if possible, let $b_{j}=0$. Then $x_{j}$ is stochastically independent of $\Sigma b_{i} x_{i}$ and so from Theorem $1 x_{j}$ is independent of the parameter $\theta$ which contradicts the assumption that the d.f. of $x_{j}$ is $F_{j}\left(x_{j}-\theta\right)$. Again, we can take all the $a_{i}$ 's different from zeros. Thus, the two linear functions $\Sigma a_{i} x_{i}$ and $\Sigma b_{i} x_{i}$ (with non-zero coefficients) of the independent random variables $x_{1}, x_{2}, \ldots, x_{n}$, are stochastically independent. Therefore, $\dagger$ all the $x_{i}$ 's must be normal variables. We thus have the following:

Theorem 3: If $x_{1}, x_{2}, \ldots, x_{n}$, are independent random variables such that their joint d.f. involves an unknown location parameter $\theta$ then a necessary and sufficient condition in order that $\Sigma b_{i} x_{i}$ is a boundedly complete sufficient statistic for $\theta$ is that $b_{i}>0$ and that $x_{i}$ is a normal variable with mean $\theta$ and variance $b_{i}^{-1}(i=1,2, \ldots, n)$.

Let us now turn to the case of the Gamma variables. Let $x_{1}, x_{2}, \ldots, x_{n}$, be independent Gamma variables with the same scale parameter $\theta>0$, i.e., the density function of $x_{i}$ is

$$
f_{i}(x) d x=\frac{1}{\Gamma\left(m_{i}\right) \theta^{m_{i}}} x^{m_{i}-1} e^{-x / \theta} d x \quad\left(x \geqslant 0, \quad \theta>0, \quad m_{i}>0\right) .
$$

It is clear then that $\Sigma x_{i}$ is a sufficient statistic for $\theta$ and its completeness follows from the unicity property of the Laplace transform. Thus, we at once have the well known result that $\Sigma x_{i}$ is stochastically independent of any function $f\left(x_{1}, x_{2}, \ldots, x_{n}\right)$ that is independent of changè of scale (i.e. independent of $\theta$ ).

Recently it has been proved by R. G. Laha that if $x_{1}, \ldots, x_{n}$, are independent and identically distributed chance variables and if $\Sigma x_{i}$ is independent of $\Sigma a_{i j} x_{i} x_{j} /\left(\Sigma x_{i}\right)^{2}$ then (under some further assumptions) all the $x_{i}$ 's must be Gamma variables. Using this result we can immediately get a characterization of the Gamma distribution analogous to Theorem 3 .

\section{REFERENCES}

Basu, D. (1951): On the independence of linear functions of independent chance variables. Bull. Int. Stat. Inst., 33, Pt. 2, 83-96.

Darmors, G. (1951): Sur diverses proprietes characteristiques de la loi de probabilito de Laplace-Gauss, Bull. Int. Stat. Inst., 33, Pt. 2, 79-82.

(1953) : Analyse generale des liaisons stochastiques-Etude particuliere de l'analyse factorielle lineaire. Rev. Inst. Internat. Stochastique, 21, 2-8.

Halmos, P. R. ANd Savage, L. I. (1949): Application of the Radon-Nikodym theorem to the theory of sufficient statistics. Ann. Math. Stat., 20, 225-241.

Lehmann, E. L. AND Scheffé, H. (1950) : Completeness, similar regions and unbiased estimation. Sankhya $, 10,305 \cdot 340$.

LAHA, R. G. (1954): On a characterization of the Gamma distribution. Ann. Math. Stat., 25, 784-787.

+ This result was first conjectured (and proved under certain assumptions) by the author in 1951. The proof without any assumption is due to G. Darmois (1953). 